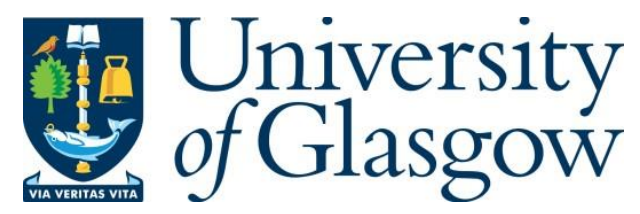

Di Campli San Vito, P., Brewster, S., Pollick, F., Thompson, S., Skrypchuk, L. and Mouzakitis, A. (2020) Purring Wheel: Thermal and Vibrotactile Notifications on the Steering Wheel. In: 22nd ACM International Conference on Multimodal Interaction, Online Only, 25-29 Oct 2020, pp. 461-469. ISBN 9781450375818 (doi: $\underline{10.1145 / 3382507.3418825}$ ).

There may be differences between this version and the published version. You are advised to consult the publisher's version if you wish to cite from it.

(C) Association for Computing Machinery 2020. This is the author's version of the work. It is posted here for your personal use. Not for redistribution. The definitive Version of Record was published in Proceedings of the 22nd ACM International Conference on Multimodal Interaction, Online Only, 25-29 Oct 2020, pp. 461-469. ISBN 9781450375818.

\title{
http://eprints.gla.ac.uk/222883/
}

Deposited on: 07 September 2020

Enlighten - Research publications by members of the University of Glasgow http://eprints.gla.ac.uk 


\section{Purring Wheel: Thermal and Vibrotactile Notifications on the Steering Wheel}

\author{
Patrizia Di Campli San Vito* \\ p.di-campli-san- \\ vito.1@research.gla.ac.uk \\ School of Computing Science, \\ University of Glasgow \\ Glasgow, United Kingdom \\ Simon Thompson \\ sthom261@jaguarlandrover.com \\ Jaguar Land Rover Ltd. \\ Coventry, United Kingdom
}

\author{
Stephen Brewster \\ stephen.brewster@glasgow.ac.uk \\ School of Computing Science, \\ University of Glasgow \\ Glasgow, United Kingdom
}

\author{
Lee Skrypchuk \\ lskrypchuk@jaguarlandrover.com \\ Jaguar Land Rover Ltd. \\ Coventry, United Kingdom
}

\author{
Frank Pollick \\ frank.pollick@glasgow.ac.uk \\ School of Psychology, University of \\ Glasgow \\ Glasgow, United Kingdom
}

\author{
Alexandros Mouzakitis \\ amouzak1@jaguarlandrover.com \\ Jaguar Land Rover Ltd. \\ Coventry, United Kingdom
}

\begin{abstract}
Haptic feedback can improve safety and driving behaviour. While vibration has been widely studied, other haptic modalities have been neglected. To address this, we present two studies investigating the use of uni- and bimodal vibrotactile and thermal cues on the steering wheel. First, notifications with three levels of urgency were subjectively rated and then identified during simulated driving. Bimodal feedback showed an increased identification time over unimodal vibrotactile cues. Thermal feedback was consistently rated less urgent, showing its suitability for less time critical notifications, where vibration would be unnecessarily attention-grabbing. The second study investigated more complex thermal and bimodal haptic notifications comprised of two different types of information (Nature and Importance of incoming message). Results showed that both modalities could be identified with high recognition rates of up to $92 \%$ for both and up to $99 \%$ for a single type, opening up a novel design space for haptic in-car feedback.
\end{abstract}

\section{CCS CONCEPTS}

\section{- Human-centered computing $\rightarrow$ Haptic devices.}

\section{KEYWORDS}

Thermal; vibrotactile; haptic; in-car; feedback

ACM Reference Format:

Patrizia Di Campli San Vito, Stephen Brewster, Frank Pollick, Simon Thompson, Lee Skrypchuk, and Alexandros Mouzakitis. 2020. Purring Wheel: Thermal and Vibrotactile Notifications on the Steering Wheel. In Proceedings of the 2020 International Conference on Multimodal Interaction (ICMI '20), October 25-29, 2020, Virtual event, Netherlands. ACM, New York, NY, USA, 9 pages. https://doi.org/10.1145/3382507.3418825

\section{INTRODUCTION}

Notifying users through vibration has become an everyday occurrence due to its use in smartphones, game controllers and even cars. Some manufacturers use it as lane departure warning [3], which

${ }^{*}$ Main Author has been shown to be effective even without prior training on the warning [30] and to have reduced the number of crashes of all severities by $18 \%$ [3]. Tactile cues, additionally, have advantages over auditory feedback in noisy environments [19] and visual cues in critical driving scenarios [24]. In multimodal use, combined with audio and/or visual cues, the addition of tactile warnings decreased reaction time [10,18, 24] and improved the driving experience [15].

The number of variations of different vibrotactile warnings is, however, limited. While the use of Tactons (tactile icons) was shown to be very effective for the single parameters roughness (recognition rate of $80 \%$ ) and rhythm (93\%), the recognition dropped to $71 \%$ [2] when these were combined. The addition of a second type of tactile feedback, using a different perceptual channel to vibration, could aid the differentiation of stimuli and not only broaden the design space of tactile feedback, but also increase recognition rates.

Thermal feedback is such a second type of tactile feedback, addressing other sensory receptors than vibration. Research around thermal stimulation has increased and its characteristics have been explored for thermal displays $[12,13]$ and several use cases $[9,16,21$, 34]. For example, the direction of temperature change (warm/cool) could be detected with an accuracy of $97.4 \%$ [32]. Additionally, thermal feedback can be associated with emotions [1, 17, 20, 29, 33], a characteristic which could add an implicit meaning to feedback, for example, increasing or decreasing the feeling of danger or urgency. The interpretation of thermal stimuli is highly dependent on the exact application, but this natural mapping can have strong associations. Many cars already have heated steering wheels and seats, so the concept of temperature changes occurring at those locations is known to drivers. As humans feel temperature changes rather than specific discrete temperatures, the feedback itself can be based on any temperature setting the driver chose for their comfort. Furthermore, thermal feedback during driving has already been investigated for directional information [5-7] with recognition rates up to $97 \%$. The combination of temperature change and vibration has not yet been tested in a vehicular environment, where recognition of notifications becomes a secondary task to the highly demanding primary task of driving.

Therefore, we designed two experiments to explore the implications of combining thermal and vibrotactile feedback for in-car 
notifications, a purring steering wheel. The first investigated how the perceived urgency of notifications would be influenced by the combination, presenting them simultaneously, and how the modalities compare to each other. In the second study, notifications with two types of information were presented either unimodally with thermal feedback only or bimodally, combining thermal and vibrotactile cues. The main contributions of this research are:

- We show the perceived urgency and identification rates of thermal, vibrotactile and bimodal tactile feedback as secondary task in a simulated driving scenario;

- We found that thermal feedback was rated as less urgent than both vibrotactile and bimodal tactile feedback and bimodal tactile feedback took longer to identify than vibration;

- We found that the association of thermal feedback with certain information can be stronger than vibration and can be used uniand bimodally to convey complex information.

\section{RELATED WORK}

\subsection{Vibrotactile Feedback in the Car}

Research into the use of haptic feedback in cars is plentiful. Several studies have investigated haptic feedback on the steering wheel mostly for navigation $[4,15,22]$ and lane departure warnings [3, 30].

For example, Ege et al. [8] enhanced auditory navigation cues with vibration on the steering wheel in noisy environments. They found that bimodal presentation led to fewer navigation errors and subjective comments indicated that the perceived level of performance was increased in the bimodal condition, as participants felt reassured by the tactile feedback.

Sadeghian Borojeni et al. [25] used vibration on the steering wheel to aid drivers of partially autonomous cars after take-overs. They found that vibration right after the take-over request did not influence the decision making, but again reassured participants and decreased their perceived mental workload.

Vibration patterns on the steering wheel for directional cues and alerts were investigated by Hwang et al. [11], combining spatial and temporal patterns. They tested different alert durations and found high recognition rates above $85 \%$, showing that vibrotactile feedback can be effectively distinguished when presented with different durations.

Suzuki et al. [30] presented vibration and acoustic alerts for lane change warnings, without having introduced the feedback to the participants beforehand. The reaction time with vibrotactile warnings was highly reduced as compared to audio. When the feedback was introduced first, audio warnings outperformed haptic feedback. Nonetheless, this study shows that tactile feedback can present certain information in a more implicit way than acoustic signals.

Vibrotactile cues for other warnings during driving have often been investigated in multimodal settings and not on the steering wheel. Kaltenberger et al. [14] presented vibration on the seat and seat belt to influence the driving style towards less $\mathrm{CO} 2$ consumption. They showed that the stimuli on the seat belt achieved this goal without explaining the purpose of the vibration beforehand.
The same result was not seen for the vibration on the seat, showing that the implicit vibrotactile feedback might be influenced by factors such as location.

Comparing tactile, visual and auditory warnings for rear-end collision prevention, Scott and Gray [27] found that drivers reacted faster to vibrotactile than visual warnings. The vibrations were presented on the waist, simulating a seat belt.

Politis et al. [23] investigated multimodal warnings with differing levels of urgency, combining vibrotactile, audio and visual cues. The vibration patterns differed in length and number of bursts. They found that participants reacted faster to more urgent warnings and also to multimodal ones. Specifically, they found that visual warnings slowed the response time in critical situations. Their vibrotactile warning design was adapted for the study presented in this paper.

This existing research shows that vibrotactile feedback can decrease perceived mental workload and reaction time in bimodal settings and can have high recognition rates even when used unimodally.

\subsection{Thermal Feedback}

The combination of vibrotactile feedback with thermal cues as a second tactile modality has been explored outside of driving.

Singhal et al. [28] investigated how thermal and vibrotactile feedback could be distinguished when presented at the same time. They found that the presentation of vibrotactile sensations can influence the ability to distinguish thermal patterns. Warm stimuli where recognised with higher accuracy than cool.

Also combining vibrotactile with thermal feedback, Wilson et al. [31] investigated emotional associations. The grouping of thermal and vibrotactile stimuli showed that vibration defined the arousal of the stimulus while thermal influenced the valence: cool temperatures lead to unpleasant/low valence and warm temperatures to pleasant/high valence associations. These findings suggest an association of cool temperatures with negative occurrences such as danger. Another study investigating user interpretations of temperature changes to indicate the level of security of web pages showed that warmer temperatures were clearly associated with dangerous pages [35]. These findings seem contradictory and show that inherent interpretation of thermal feedback is highly dependent on the application.

Salminen et al. [26] investigated the emotional responses to thermal stimuli and found that warmth elevated both dominance and arousal, suggesting that the addition of warm stimuli to vibration could elevate the perceived level of urgency of a warning.

In all these experiments the detection of thermal feedback was the main challenge. During driving, however, the recognition becomes a secondary task alongside a highly demanding primary task, which could influence the recognition further and needs additional examination.

We, see Di Campli San Vito et al. [5, 6], investigated the use of thermal feedback for lane change scenarios during driving, indicating the direction of desired lane change by the direction of temperature change (warm/cold). The recognition rate for thermal feedback was high, but participants rated it as being more mentally demanding and frustrating than speech. However, participants in the study had to drive one-handed, as the thermal feedback was 


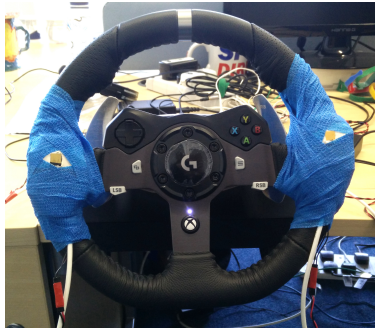

(a) Steering Wheel of first experiment

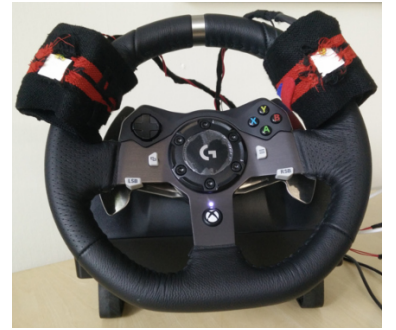

(b) Steering Wheel of second experiment
Figure 1: Steering wheels equipped with hardware: Peltier elements and vibrotactile actuators on each side.

presented only by a single thermal device on a table, which they touched with their right hand.

In further studies, we investigated thermal feedback on the steering wheel for navigation [7]. Thermal feedback during driving had recognition rates up to $97 \%$, but the presentation of warm and cool stimuli on different hands at the same time led to confusion. In the experiment presented in this paper the thermal device elements were also attached to the steering wheel, but both sides showed the same thermal feedback.

The user studies in this paper were informed by this previous research. Thermal and vibrotactile feedback were tested individually and together during simulated driving, with the devices being attached on the steering wheel, allowing comfortable and stable driving.

\section{APPARATUS}

Thermal feedback was given by thermoelectric Peltier devices that were attached to a heat-sink and mounted on a Logitech G920 Driving Force Racing Wheel, one on the left and one on the right side. In the first experiment, $1 \times 1 \mathrm{~cm}$ Peltier devices (Figure 2(b), upper right) were attached with elastic bandages (see Figure 1(a)), in the second study $2 \times 2 \mathrm{~cm}$ devices (Figure $2 \mathrm{~b}$, bottom right) were attached using sport bands (see Figure 1(b)). In both experiments, they were accompanied by one Haptuator Mark II vibrotactile actuator each (see Figure 2(b), upper right), providing the vibration. The steering wheel was connected to a Dell XPS Windows laptop, as were Sennheiser HD 25 Basic Edition headphones and a BENQ DLP projector. The projected driving scenario was implemented with OpenDS 3.5 free version ${ }^{1}$. The participants were seated in a gaming racing chair. The complete set-up is presented in Figure 2(a).

\section{EXPERIMENT 1: DIFFERENT LEVELS OF URGENCY}

\subsection{Study Design}

The experiment was designed as a $5 \times 3$ within-subjects study with modality (5 levels: Bimodal Warm, Bimodal Cold, Thermal Warm, Thermal Cold and Vibration) and level of urgency (3 levels: high, medium and low) as the Independent Variables. The Dependent

\footnotetext{
${ }^{1}$ https://opends.dfki.de/ (accessed 20/05/2020)
}

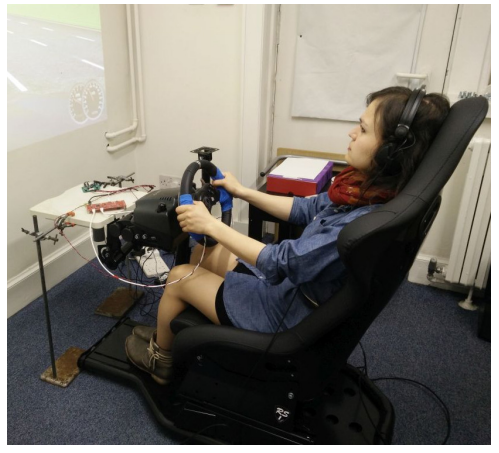

(a) Set-up of the experiment

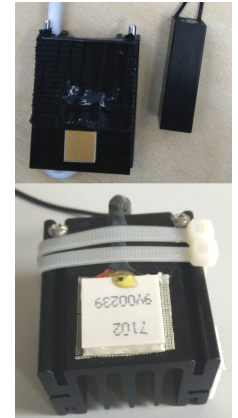

(b) Peltiers and vibrotactile Haptuator
Figure 2: Set-up (a) and hardware (b): Peltiers (golden and white squares) on the black heat sinks and vibrotactile Haptuator (black bar)

Variables were subjective urgency rating, urgency identification accuracy, recognition time and subjective feedback.

We hypothesised that the combination of thermal and vibroctactile feedback would increase the perceived urgency of the notifications. Due to the less immediate nature of thermal feedback in the temperature range we used, its perceived urgency was also expected to be lower than vibrotactile feedback. As research has shown that bimodal representations of warnings increased the recognition rate [8], we expected this to be the case here as well. However, because thermal feedback takes longer to be detected, compare [6, 7], the recognition was expected to take longer for thermal feedback.

The vibrating patterns were adapted from Politis et al. in [23]. They encoded the different levels of urgency into vibration patterns, adapting the length and number of individual vibrations. Each stimulus was around $1.5 \mathrm{~s}$ long and all stimuli were $250 \mathrm{~Hz}$. The urgent notification consisted of eight $0.1 \mathrm{~s}$ pulses with $0.1 \mathrm{~s}$ intervals (see Figure 3(a)), the medium notification had five pulses of $0.17 \mathrm{~s}$ with intervals of $0.17 \mathrm{~s}$ (resulting in a stimuli length of $1.53 \mathrm{~s}$, see Figure $3(\mathrm{~b})$ ) and the low urgency notification consisted of two pulses of $0.5 \mathrm{~s}$, with an interval of $0.5 \mathrm{~s}$ (compare Figure 3(c)). The thermal feedback was designed to match this overall time frame, ensuring the initial temperature change could be fulfilled parallel to the vibration. The highest round number temperature that the hardware could reach within $1.5 \mathrm{~s}$ was $4^{\circ} \mathrm{C}$ with a rate of change of $3^{\circ} \mathrm{C} / \mathrm{s}$. We decided on $4^{\circ} \mathrm{C}$ rather than $4.5^{\circ} \mathrm{C}$ to ensure at least a short period in which the temperature was kept constant, as literature has shown that the recognition of thermal warm stimuli without a period of constant temperature was negatively impacted $[5,6]$. The base temperature was set to $30^{\circ} \mathrm{C}$, so the highest and lowest temperatures were $34^{\circ} \mathrm{C}$ and $26^{\circ} \mathrm{C}$, respectively. These were used for the urgent notifications. The medium stimuli warmed and cooled $2^{\circ} \mathrm{C}$, the low ones $1^{\circ} \mathrm{C}$. These temperatures were chosen to ensure that the urgent cues, the most important one, would be the easiest to distinguish. As the lower temperature changes were very subtle, we wanted to explore how well they could be felt and distinguished. The return to the neutral temperature occurred after $1.5 \mathrm{~s}$ with a 


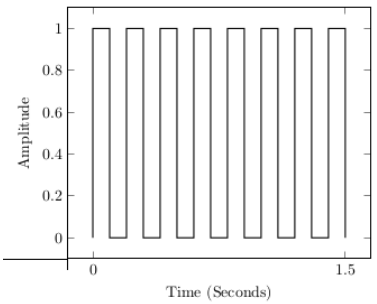

(a) Vibration High Urgency

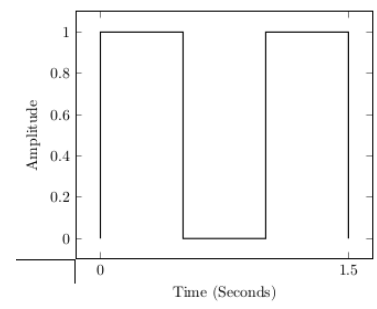

(c) Vibration Low Urgency

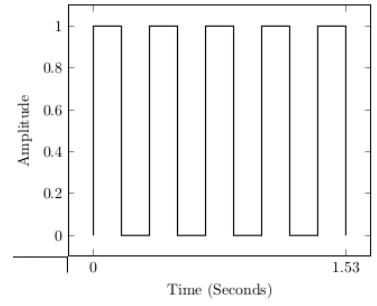

(b) Vibration Medium Urgency

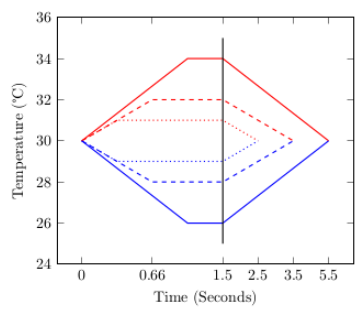

(d) Thermal Changes
Figure 3: Vibration Patterns and Thermal Feedback for Experiment 1: solid line for high, dashed line for medium and dotted line for low urgency notifications. The time axis is contracted for the time of the return to neutral. The vertical black line indicates the end of the vibration stimulus.

rate of change of $1^{\circ} \mathrm{C} / \mathrm{s}$ to decrease false positive recognitions at the return to neutral $[5,6]$ (compare Figure 3(d)). In the bimodal conditions, the thermal and vibrotactile notifications started at the same time. The return to the neutral temperature therefore occurred when the vibration pattern ended.

4.1.1 Part 1: Subjective Rating. The experiment was divided into two parts: first, the participants were asked to subjectively rate the perceived level of urgency of the feedback with a slider, from values between 0 and 100 . The stimuli were not described to the participants in detail beforehand, they were only told to expect temperature changes, vibration or a combination of the two. The participants placed their hands on the steering wheel and moved the slider left and right by turning the wheel. Small movements in the corresponding direction steadily and slowly moved the slider, bigger movements increased the speed. The ratings were submitted when participants pulled either of the gear paddles behind the steering wheel. Participants were asked to rate 0 if they did not perceive a stimulus within $5 \mathrm{~s}$. As the low and medium urgency notifications were very subtle and therefore might be missed, this was introduced to eliminate long pauses and enable filtering of the missed notifications. The stimuli were presented randomly within $3 \mathrm{~s}$ after the submission of the previous rating. Each stimulus was presented 3 times in this task, resulting in 45 stimuli overall. Headphones played white noise throughout this part of the experiment, covering any noise from the vibrotactile actuators.

4.1.2 Part 2: Recognition during driving. The second part of the study started with an introduction to the different stimuli. Every notification was shown to the participants twice, with the experimenter pointing out the different levels of urgency and making sure the participant could feel and distinguish all notifications. They were asked to indicate the level of urgency presented by the stimulus by pressing a button on the steering wheel: the top button represented urgent notifications, the middle button medium notifications and the lowest button the low notifications. They were then introduced to the driving task, utilising lane changes to keep the driver engaged. The lane changes were initiated visually by presenting arrows above the lane the participant should change into. These arrows appeared on bridges ahead and the participants were asked to change into the lane before they reached the signs. Between each lane change, one notification was presented randomly within a time frame of $3 \mathrm{~s}$. The lane changes and the notifications did not overlap. The stimuli were chosen randomly, as were the destination lanes. The whole procedure was first explained to the participants and then followed by a training session with 3 stimuli (urgentBimodalCold, mediumThermalWarm, lowVibration) and lane changes presented to them during driving. The main driving task followed and consisted again of 45 stimuli, 3 presentations of each stimulus. The driving was divided into 5 blocks of 9 notifications each. The participants heard driving noises generated by the simulator through the headphones throughout driving.

After driving, participants filled in a questionnaire collecting demographic data and qualitative data on the feedback types. The study was approved by our institution's Ethics Committee.

\subsection{Participants and Procedure}

Eighteen participants ( 10 female, 8 male) between 19 and 48 years (Mean=27.56, $S D=7.59$ ) completed the experiment. No uncorrected vision and sensory impairments of the hands were reported. The driving experience ranged from 0 to 30 years (Mean=8.89, $S D=7.47$ ). The participants rated prior experience with driving simulators (Median=2), vibrotactile feedback (Median=2) and thermal feedback $($ Median $=1)$ on a 5 point Likert scale (1 equalled none, 5 very much experience).

On arrival, participants were presented with an information sheet explaining the experiment and were then asked to sign a consent form. They were seated in the gaming chair and completed the two parts of the study. At the end of the experiment the participants filled in a questionnaire collecting demographic data and feedback rankings. The study was completed within one hour and the participants received $£ 6$.

\subsection{Results}

Statistical values of post hoc tests, calculated with Bonferroni corrections, had to be omitted in this paper for the sake of brevity ${ }^{2}$. Some results had to be corrected with Greenhouse-Geisser, as sphericity was violated.

Bimodal and thermal stimuli include both warm and cold stimuli, if the direction of change is not specifically mentioned.

4.3.1 Subjective Urgency Rating of Part 1. The subjective ratings at the beginning of the experiment, see Figure 4, were evaluated using

${ }^{2}$ All statistical results can be found at

http://dx.doi.org/10.5525/gla.researchdata.1054 


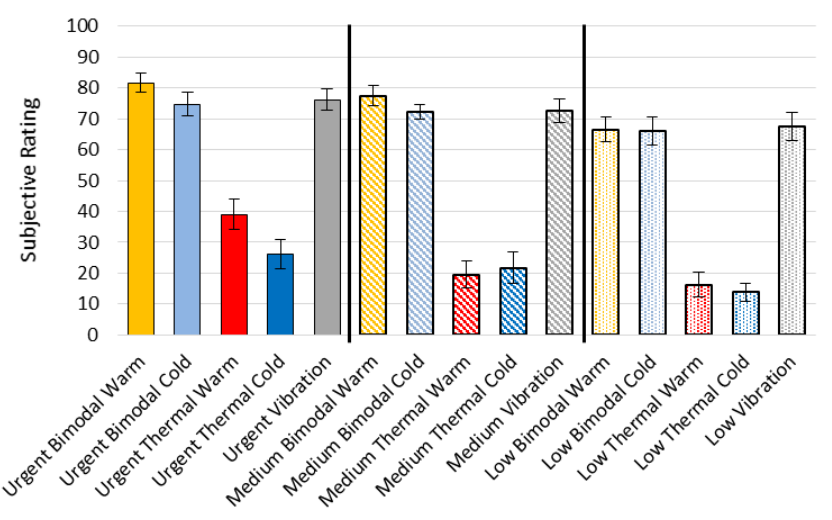

Figure 4: Subjective Ratings from Part 1, error bars show the standard error on all graphs.

a repeated measures ANOVA. Urgency was found to have a statistically significant influence on the subjective rating $(F(2,20)=12.56$, $p<0.001)$, as did Modality $(F(1.91,19.13)=36.00, p<0.001)$. However, as the interaction of the two factors showed significant differences as well $(F(4.20,41.98)=3.65, p=0.01)$, those will be discussed as they provide more detailed results. Post hoc tests showed significant differences between bimodal and vibrotactile conditions with all low and medium thermal conditions and UrgentThermalCold. These thermal cues were rated as less urgent. There were no differences between bimodal and vibrotactile conditions for any urgency. UrgentThermalWarm was found to be only significantly different from bimodal warm cues of all urgencies (rated as less urgent), as well as low and medium thermal warm cues and LowThermalCold (rated as more urgent). All stimuli rated 0 (not noticed) were compared with a repeated measures ANOVA and showed a significant difference for urgency $(F(2,34)=13.21, p<0.001)$, Modality $(F(4,68)=15.73$, $p<0.001)$ and their interaction $(F(8,136)=6.01, p<0.001)$. Post hoc tests for the interaction Bimodal and vibration were significantly different from low and medium thermal cues of all urgencies. In addition, UrgentThermalCold was different from vibrotactile cues of all urgencies, medium and urgent bimodal cues both warm and cold changes as well as LowBimodalCold and LowThermalCold. UrgentThermWarm was also different from LowThermalCold, as well as low and medium thermal warm cues.

4.3.2 Urgency Identification of Part 2. The recognition rates can be seen in Figure 5. The recognition accuracy of the stimuli in Part 2 was evaluated with a repeated measures ANOVA and showed no significant differences for Urgency $(F(2,34)=2.48, p=0.10)$, but for Modality $(F(2.04,34.73)=21.41, p<0.001)$ and the interaction of the factors $(F(3.99,67.74)=3.91, p=0.007)$. Post hoc tests of the interaction showed significant differences between the urgent thermal cues and bimodal and vibrotactile cues of all urgencies, where thermal was recognised correctly less often. For low and medium thermal cues, there were more differences found for warm temperature changes than cold. While MediumThermalCold was only found to be different from urgent bimodal and vibrotactile cues, the warm counterpart was additionally different from LowBimodalCold, and medium bimodal and vibrotactile cues. LowThermalCold was only

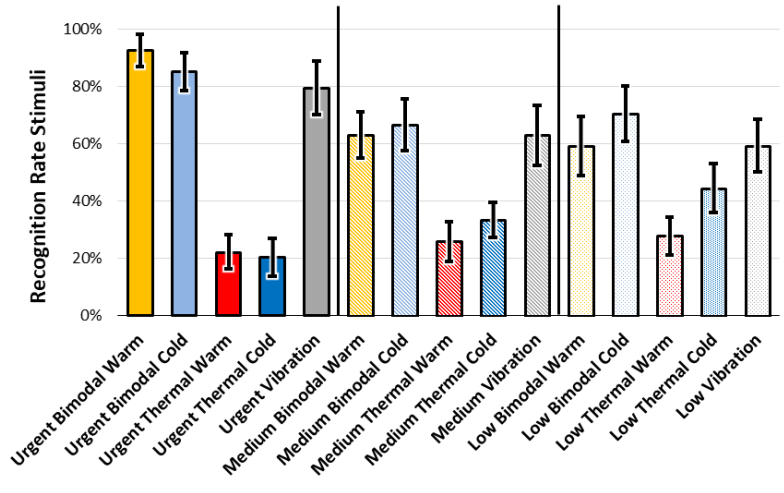

Figure 5: Urgency Recognition Rates for All Stimuli.

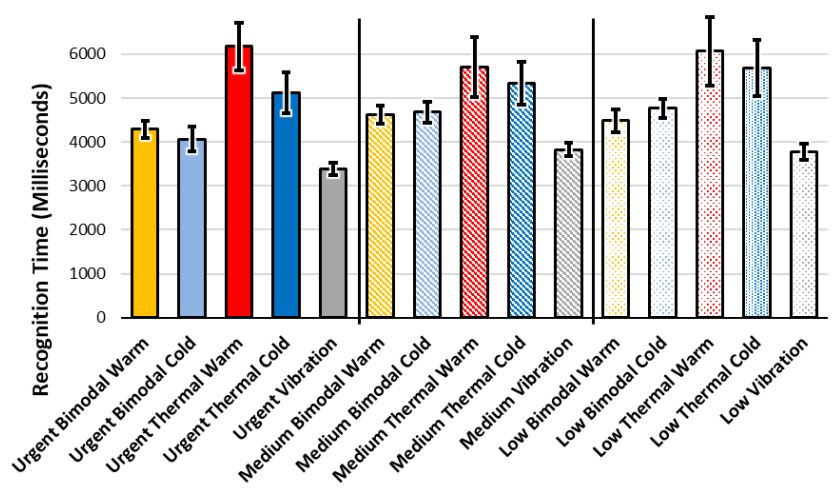

Figure 6: Recognition Time for Urgencies and Modalities.

different from the urgent bimodal cues, while LowThermalWarm was additionally different from MediumBimodalCod and UrgentVibration. Cold temperature changes were recognised easier than warm, but less effectively than bimodal or vibrotactile cues. Some of the thermal stimuli were not rated at all, indicating that participants had problems distinguishing the urgencies or had problems feeling the feedback. The number of unrated stimuli was higher for warm than for cold temperatures and increased with lower urgency.

4.3.3 Recognition Time. The recognition time of the second part of the experiment was evaluated with a repeated measures ANOVA and showed statistically significant differences for urgency $(F(2,22)=12.33$, $p<0.001)$. Post hoc tests showed significant differences for urgentmedium as well as urgent-low, but not medium-low. Urgent notifications were on average recognised $258 \mathrm{~ms}$ faster than medium and $290 \mathrm{~ms}$ faster than low notifications.

Modality showed a significant difference $F(2.02,22.22)=18.80$, $p<0.001)$. Post hoc tests showed statistically significant differences for bimodal-thermal warm, bimodal-vibration and thermal-vibration, see Figure 6. Vibrotactile notifications were recognised faster than any other modality, while recognition of thermal warm notifications took the longest.

4.3.4 Subjective Feedback. The evaluation of the questionnaire at the end of the experiment showed that most participants preferred 
the vibrotactile condition (10, compared to 8 who voted for bimodal; none preferred thermal).

Participants were asked to comment on the reasons for their preference and most wrote that vibration was easier to distinguish than the thermal differences. One participant wrote when combined with vibration, the temperature changes made the overall notification feel more urgent and this sentiment was shared by 4 others. Overall, 11 participants rated the bimodal condition as the most urgent feedback type. However, several participants commented on some uncertainty connected with thermal feedback, while vibrotactile feedback was described as clear and easy to identify.

When asked which direction of temperature felt more urgent, 12 participants named warm and 5 cold, 1 described both as equally urgent. Furthermore, 8 participants described warm as more comfortable, another 8 cold and 2 found both equally comfortable.

Additionally, participants rated on a 5-point Likert scale (with the option to rate I don't know, which was treated as 0 on the scale), how well they could feel the warm and cool stimuli alone and when presented with vibration. The additional 0 option was added for cases in which participants had problems feeling a specific stimulus. saying that they were not sure whether the warm feeling when holding the steering wheel was a stimulus or the hand warming up the steering wheel. The ratings for the bimodal representations were higher (warm: Median=4.5; cold: Median=4) than for thermal alone (warm: Median=3; cold: Median=2).

\subsection{Discussion}

When asked directly which modality felt most urgent in the questionnaire at the end, most participants chose the bimodal conditions, however, this was not mirrored in the subjective rating at the beginning of the experiment. While bimodal and vibrotactile cues showed no difference in perceived urgency, it was quite pronounced between thermal and all other conditions: thermal was rated consistently less urgent.However, the number of missed stimuli was quite high, especially for the low urgencies, which showed that perception of these very subtle temperature changes was difficult.

The urgency identification was better for bimodal and vibrotactile feedback than thermal. It was expected that bimodal cues would be higher than vibration, but this was not observed.

The evaluation of the recognition times showed that the warm thermal cues took significantly longer to be identified than bimodal and vibrotactile ones. However, the cold thermal stimuli were only significantly slower than vibration and not bimodal stimuli.

Surprisingly, bimodal feedback took longer to be recognised than vibration. It would seem that the addition of thermal stimuli slowed the fast response time of vibration. This, in addition to the high number of missed stimuli for thermal feedback and the fact that the bimodal condition only slightly increased the perceived level of urgency, makes this bimodal feedback design unsuitable for urgent notifications that require fast responses. The thermal feedback, designed to fit the short times of the notification design by Politis et al., seemed to be too subtle for effective recognition. Longer notifications with higher temperature changes might prove more effective.

Participants did comment on a increased feeling of urgency when presented with bimodal cues. These results could be beneficial in designing haptic feedback for grouped notifications: Vibration patterns could, for example, define one aspect (high/low importance) or the type (message/navigation/fuel alert) of a notification, while thermal feedback could be used to assign information to those, taking advantage of the many associations of temperature changes: personal/work message (warm/cold), change of time to destination (warm/cold mapped to longer/shorter).Additionally, the consistent rating of low perceived urgency for thermal feedback qualifies this feedback type for information which does not need immediate action and for which vibration is unnecessarily attention-grabbing.

One of the limitations of this prototype is that participants had to hold the steering wheel at specific locations, which can influence the comfort of driving. We envision the entirety of the steering wheel vibrating and changing temperatures for practical future use, which would overcome this. Furthermore, sensors on the steering wheel could measure the skin's temperature and the feedback could be directly adapted to this as the baseline, which in turn could positively influence perception and comfort. With this vision in mind, the second experiment looked into the combination of thermal and vibrotactile feedback for different types of information.

\section{EXPERIMENT 2: TWO TYPES OF INFORMATION FOR MESSAGE CLASSIFICATION}

\subsection{Study Design}

The second experiment was designed as a $2 \times 2 \times 2$ within-subjects study with Modality (thermal/bimodal (vibrotactile and thermal)), Importance (high/low) and Nature (personal/work) of message as the Independent Variables. In both modalities, the Nature of the message was communicated by the direction of temperature change (warm for personal / cool for work). The Dependent Variable were recognition rate and subjective feedback, collected with demographic data in a questionnaire at the end of the experiment.

All thermal stimuli started from a neutral temperature of $30^{\circ} \mathrm{C}$ and changed in both directions $6^{\circ} \mathrm{C}$ at $3^{\circ} \mathrm{C} / \mathrm{s}$. Importance in the thermal only condition (T-Importance) was differentiated by the intensity of the stimulus, a combination of how long the temperature was presented and how fast it returned to neutral. The different thermal stimuli were chosen from the set of combinations investigated previously [5, 6]: they all showed recognition rates of over $90 \%$, while false recognitions at the return to the neutral temperatures were under $10 \%$. The high importance thermal notification was presented for $3 \mathrm{~s}$ and then returned to the neutral temperature slowly by changing the temperature $1^{\circ} \mathrm{C}$ at a rate of $1^{\circ} \mathrm{C} / \mathrm{s}$, then holding this temperature for $1 \mathrm{~s}$ before changing again. This process was repeated until the neutral temperature was reached (see Figure 7, solid line). The low importance thermal stimulus returned to the neutral temperature directly at $1^{\circ} \mathrm{C} / \mathrm{s}$, see Figure 7 dotted line. The thermal part of the bimodal notification is presented by the dashed line in Figure 7: the temperature was kept for $3 \mathrm{~s}$ before returning to the neutral temperature at $1^{\circ} \mathrm{C} / \mathrm{s}$. The two vibration patterns encoding Importance (V-Importance) in the bimodal condition were adopted from Experiment 1: the low urgency pattern (see Figure 3(c)) was used for the low importance notifications, while the high urgency pattern (Figure 3(a)) encoded high importance. Ample research has investigated aspects of vibrotactile feedback to convey 


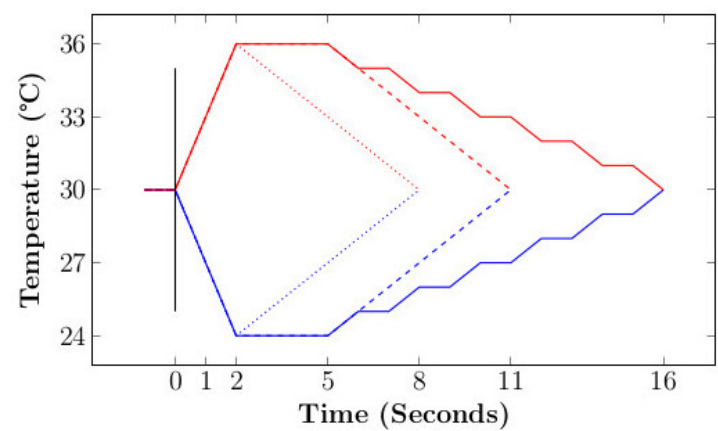

Figure 7: Thermal Changes of Experiment 2: solid line for high importance thermal stimuli; dotted line for low importance thermal stimuli; dashed line for thermal part of bimodal stimuli. The time axis is contracted for the time of the return to neutral. The vertical black line indicates the end of the vibration stimulus.

multi-levelled information (i.e. Tactons [2]), therefore, we excluded vibration only cues and focused on novel thermal feedback.

We hypothesised the recognition rate for bimodal stimuli to be higher, as vibration is more attention-grabbing and widely used. As the stimuli were chosen based on their high recognition rates and low rates of false positives, a similar result was expected for the thermal feedback in this experiment.

The driving scenario was similar to the one adopted in Experiment 1. Participants were asked to change lanes when indicated by the arrows on the bridges and the stimuli were presented between the changes. The 8 different stimuli as combinations from the Independent Variables were presented 5 times each. In contrast to Experiment 1, five lane changes without any stimuli present were introduced, so participants would feel confident about not feeling a stimulus and would not feel pressured into reporting anything when they were unsure. This resulted in 45 lane changes overall with 40 presented stimuli. These were chosen randomly during driving and presented in five driving blocks of 9 changes each. Participants orally reported the importance and nature of the notifications to the experimenter. This ensured that the hands were kept on the devices throughout the experiment and the driving was not influenced by the button pressing process, but as a result recognition times could not be extracted. The study design was approved by our institution's Ethics Committee.

\subsection{Participants and Procedure}

Eighteen newly recruited participants ( 8 male, 9 female, 1 nonbinary) between 18 and 36 years (Mean $=25.72, S D=4.93)$ completed the study. All reported at least corrected vision and no sensory impairments in their hands. Their driving experience ranged between 0.5 and 18 years (Mean=6.33, $S D=4.80)$ and they reported prior experience with driving simulators (Median $=2)$, vibrotactile (Median=2) and thermal (Median=1) feedback on a 5-point Likert scale as in the previous experiment.

Participants were presented with the information sheet, followed by a consent form to sign. The participants then started the experiment. At the end, participants filled in a questionnaire capturing

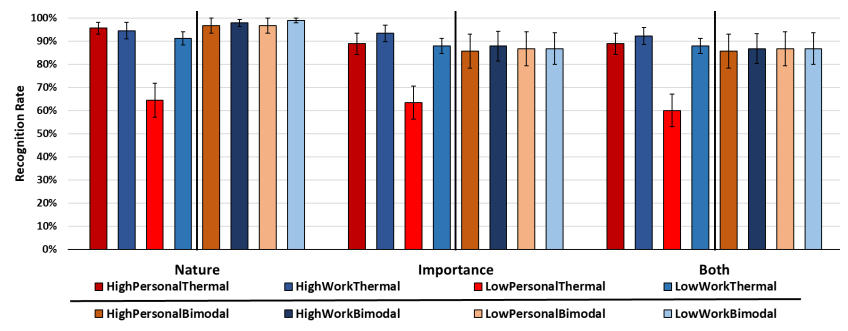

Figure 8: Recognition rates for Nature, Importance and both.

demographic data and ratings. The study was completed in under an hour and participants were paid by a $£ 10$ Amazon voucher.

\subsection{Results}

5.3.1 Recognition Rate. The low importance personal thermal notification (the short warm thermal only stimulus) had the lowest recognition rate (between 60 and $63 \%$ for all factors). Apart from this stimulus, the rates for Nature (warm/cold) (Figure 8 left side) were between 91 and $99 \%$ and consistently higher than Importance (vibration patterns / lengths of thermal stimuli) (Figure 8 middle, between 86 and 93\%) and both combined (Figure 8 right, between 86 and $92 \%$ ). Recognition was evaluated using repeated measures ANOVA. Results can be seen in Table 1 and show significant results for the factors Importance and Nature and all interactions between factors, but not Modality.

Table 1: Repeated measures ANOVA results for Recognition.

\begin{tabular}{lrr}
\hline & Statisic & $\mathrm{p}$ \\
\hline Importance & $\mathrm{F}(1,17)=17.94$ & $<0.001$ \\
Nature & $\mathrm{F}(1,17)=6.60$ & 0.020 \\
Modality & $\mathrm{F}(1,17)=0.44$ & 0.514 \\
Importance * Nature & $\mathrm{F}(1,17)=15.72$ & 0.001 \\
Importance * Modality & $\mathrm{F}(1,17)=18.30$ & $<0.001$ \\
Nature * Modality & $\mathrm{F}(1,17)=7.61$ & 0.013 \\
Import * Nature * Modal & $\mathrm{F}(1,17)=8.67$ & 0.009 \\
\hline
\end{tabular}

Post hoc tests for the interaction of all factors showed that the low importance personal thermal notification was significantly different from all other stimuli. There was no significant difference found between any of the other stimuli. The interactions of Importance and Nature showed that low importance personal notifications were significantly worse than low importance work, high importance personal and high importance work. Low importance thermal feedback was also statistically significantly different from high importance thermal feedback, and personal thermal feedback from work thermal feedback.

5.3.2 Subjective Feedback. Participants rated how well they could differentiate the components of the stimuli on a 5-point Likert scale: direction of temperature change (Nature) $($ Median $=4$ ), vibration patterns (V-Importance) (Median=5) and lengths of thermal stimuli (T-Importance) $($ Median=3). Friedman's ANOVA showed significant differences $\left(\chi^{2}(2)=27.75, p<0.001\right)$. Post hoc tests showed statistically significant results for T-Importance - Nature and V-Importance 
- T-Importance, but not between Nature - V-Importance. Participants preferred bimodal to thermal feedback. P05 wrote that it was easier to identify the bimodal prompts. $P 03$ agreed, but added: Although I would say that I felt like I could identify all the bimodal stimuli equally. P08 pointed out a common sentiment among some participants: with vibration it was less likely to think I had received a message when I hadn't. Some participants mixed up the two vibration patterns, $P 11$ commented on this: The vibration pattern for low importance seems more urgent than high importance to me. The high importance one is just like a normal phone notification.

\subsection{Discussion}

The low importance personal thermal stimulus was rated and recognised significantly worse than the other stimuli. Comments made by the participants suggest that they had problems distinguishing between a short warm thermal cue and their hands getting warm from holding the steering wheel. However, no warm stimulus was reported when none was presented, suggesting that while some stimuli were not reported because of uncertainty, the natural warming of the hand during driving did not elicit additional recognitions. This negates one of the most often named concerns regarding thermal feedback on the steering wheel.

When comparing recognition rates, high importance work thermal had the highest recognition rate (92\%). Recognition of Importance in bimodal stimuli might have been influenced by participants mixing up the two vibration patterns, as suggested in participants' comments. Enabling drivers to choose or create vibration patterns, as is possible for most mobile devices, could increase the recognition rates for bimodal stimuli. The factor Nature had recognition rates over $90 \%$ (low importance personal thermal stimuli excluded), the highest being the low importance work bimodal with $99 \%$. The mapping of warm to personal and cold to work seems to have elicited a clearer association than the vibration patterns. In addition, the recognition of cold stimuli seems to have been aided by the few longer bursts of vibration of the low importance vibration pattern, as opposed to the many short bursts of the high importance one.

Both bimodal and thermal stimuli, apart from the low importance personal thermal one, were recognised and distinguished equally easily during driving, with high recognition rates for both factors individually and in combination, showing that complex information can successfully be presented with thermal and bimodal tactile feedback during driving. These results promise to be a solid foundation for a rich and diverse design space for in-car notifications with different types of information.

\section{CONCLUSION}

This paper presents two studies investigating novel thermal and vibrotactile feedback on the steering wheel during driving. Participants showed high accuracy when classifying vibrotactile and thermal feedback for incoming messages for all but one stimulus. Unimodal thermal and bimodal thermal and vibrotactile notifications for three levels of urgency were recognised better for bimodal and vibrotactile than for thermal feedback alone. However, there was no difference between bimodal and vibration, suggesting that the vibration was the main cause for the correct recognition. The recognition time for bimodal notifications was significantly longer than for vibrotactile ones. This would suggest that participants did recognise the thermal changes and reacted to them, which influenced the recognition time. The high number of missed thermal stimuli in the first experiment suggests that thermal stimuli design has to be improved for thermal only notifications. Thermal stimuli were rated as less urgent than vibrotactile and bimodal notifications. When asked directly, which modality felt more urgent, participants chose bimodal, yet this rating was not mirrored by the subjective rating of the stimuli.

These findings suggest that the addition of thermal to vibrotactile feedback for time sensitive notifications is not suitable. However, haptic feedback for notifications of not time-sensitive information seems to be promising. Our second study showed promising results for both unimodal thermal and bimodal haptic feedback for identification of incoming messages, a task which does not require immediate attention. High recognition rates for two presented types of information encoded in the cues show that thermal feedback, alone or in addition to vibration, can be successfully used to convey more complex information during driving. The relation of personal to warm and work to cool temperatures was consistently recognised with high accuracy. The cool temperature changes were more often recognised correctly in regard to both Nature and Importance combined, resulting in the highest recognition rate of $92 \%$. The highest recognition accuracy overall was achieved for the factor Nature, where the low urgency work (cool) bimodal stimulus reached $99 \%$. There are many potential applications for these cues. Uni- or bimodal haptic notifications could inform drivers about the status of the car, indicating low air pressure in the wheels or reminding the driver to check the oil. Cooling of the steering wheel could indicate that the outside temperature has fallen beneath $4^{\circ} \mathrm{C}$ and dangerous road conditions could occur, warming could point out that the handbrake has not been disengaged properly. Currently all those notifications are given by easily overlooked small visual icons, overloading the already heavily engaged visual channel. In future cars, steering wheels could be designed to present tactile feedback all over its surface and adapt the feedback according to the settings. Engaging haptic sensations instead of adding more information visually surely will result in safer and more comfortable driving.

Results of these studies clearly show that thermal feedback, in combination with vibrotactile feedback or by itself, provides a valuable design space for non-urgent notifications in cars. Thermal feedback is unique in its way of providing inherent associations to feedback and this advantage could increase the driving experience immensely.

\section{ACKNOWLEDGMENTS}

This research was supported by an EPSRC iCASE Award and Jaguar Land Rover (EP/P510506/1).

\section{REFERENCES}

[1] Moses Akazue, Martin Halvey, Lynne Baillie, and Stephen Brewster. 2016. The Effect of Thermal Stimuli on the Emotional Perception of Images. Proceedings of the 2016 CHI Conference on Human Factors in Computing Systems - CHI '16 (2016), 4401-4412. https://doi.org/10.1145/2858036.2858307

[2] Lorna M. Brown, Stephen Brewster, and Helen C. Purchase. 2005. A first investigation into the effectiveness of Tactons. Proceedings - 1st foint Eurohaptics Conference and Symposium on Haptic Interfaces for Virtual Environment and Teleoperator Systems; World Haptics Conference, WHC 2005 (2005), 167-176. https://doi.org/10.1109/WHC.2005.6 
[3] Jessica B. Cicchino. 2017. Effects of lane departure warning on police-reported crash rates. Fournal of Safety Research 66, August (2017), 61-70. https://doi.org/ 10.1016/j.jsr.2018.05.006

[4] Nathalie Cotté, Joachim Meyer, and Joseph F Coughlin. 2001. Older and Younger Drivers' Reliance on Collision Warning Systems. Proceedings of Human Factors and Ergonomics Society 45th Annual Meeting (2001), 277-280. https://doi.org/10. 1177/154193120104500402

[5] Patrizia Di Campli San Vito, Stephen Brewster, Frank Pollick, Stuart White Lee Skrypchuk, and Alexandros Mouzakitis. 2018. Investigation of Thermal Stimuli for Lane Changes. Proceedings of the 10th International Conference on Automotive User Interfaces and Interactive Vehicular Applications (2018), 43-52. https://doi.org/10.1145/3239060.3239062

[6] Patrizia Di Campli San Vito, Stephen Brewster, Frank Pollick, Stuart White, Lee Skrypchuk, and Alexandros Mouzakitis. 2019. Thermal Feedback for Simulated Lane Change Scenarios. Special Issue of the International fournal of Mobile Human Computer Interaction (IFMHCI): Recent Advances in Automotive User Interfaces and Interactive Vehicular Applications Research 11, 2 (2019), 39-57. https://doi org/10.4018/IJMHCI.2019040103

[7] Patrizia Di Campli San Vito, Gözel Shakeri, Stephen Brewster, Frank Pollick Edward Brown, Lee Skrypchuk, and Alexandros Mouzakitis. 2019. Haptic Navigation Cues on the Steering Wheel. CHI19 (2019), 1-11. https://doi.org/10.1145/ 3290605.3300440

[8] Enes Selman Ege, Furkan Cetin, and Cagatay Basdogan. 2011. Vibrotactile feedback in steering wheel reduces navigation errors during GPS-guided car driving. 2011 IEEE World Haptics Conference, WHC 2011 (2011), 345-348. https: //doi.org/10.1109/WHC.2011.5945510

[9] Martin Halvey and Graham Wilson. 2013. Perception of thermal stimuli for continuous interaction. CHI'13 Extended Abstracts ... (2013), 1587-1592. https: //doi.org/10.1145/2468356.2468640

[10] Cristy Ho, Nick Reed, and Charles Spence. 2006. Multisensory in-Car Warning Signais for Collision Avoidance. Proceedings of the Human Factors and Ergonomics Society Annual Meeting 56, 1 (2006), 1107-1114. https://doi.org/10 1518/001872007X249965.

[11] Sungjae Hwang and Jung Hee Ryu. 2010. The haptic steering wheel: Vibro-tactile based navigation for the driving environment. 2010 8th IEEE International Conference on Pervasive Computing and Communications Workshops, PERCOM Workshops 2010 (2010), 660-665. https://doi.org/10.1109/PERCOMW.2010.5470517

[12] Lynette A. Jones and Michal Berris. 2002. The psychophysics of temperature perception and thermal-interface design. Proceedings - 10th Symposium on Haptic Interfaces for Virtual Environment and Teleoperator Systems, HAPTICS 2002 (2002), 137-142. https://doi.org/10.1109/HAPTIC.2002.998951

[13] Lynette A. Jones and Hsin-ni Ho. 2008. Warm or Cool , Large or Small? The Challenge of Thermal Displays. TRANSACTIONS ON HAPTICS 1, 1 (2008), 53-70.

[14] M. Kaltenberger, A. Riener, A. Ferscha, P. Frech, and M. Hackl. 2010. Subliminal vibro-tactile based notification of $\mathrm{CO} 2$ economy while driving. AutomotiveUI (2010), 92. https://doi.org/10.1145/1969773.1969790

[15] Dagmar Kern, Paul Marshall, Eva Hornecker, Yvonne Rogers, and Albrech Schmidt. 2009. Enhancing navigation information with tactile output embedded into the steering wheel. Lecture Notes in Computer Science (including subseries Lecture Notes in Artificial Intelligence and Lecture Notes in Bioinformatics) 5538 LNCS (2009), 42-58. https://doi.org/10.1007/978-3-642-01516-8\{_\}5

[16] Sven Kratz and Tony Dunnigan. 2016. ThermoTouch : Design of a High Dynamic Temperature Range Thermal Haptic Display. CHI Extended Abstracts on Human Factors in Computing Systems (2016), 1577-1582. https://doi.org/10.1145/2851581 2892554

[17] Wonjun Lee and Youn-kyung Lim. 2010. Thermo-Message: Exploring the Potential of Heat as a Modality of Peripheral Expression. Proceedings of the 28th of the international conference extended abstracts on Human factors in computing systems - CHI EA '10 (2010), 4231. https://doi.org/10.1145/1753846.1754131

[18] Penaranda B. N. Roberts D. M. \& Baldwin C. L. Lewis, B. A. 2013. Effectiveness of bimodal versus unimodal alerts for distracted drivers. Human Factors in Driver Assessment, Training and Vehicle Design (2013), 376-382.

[19] Atsuo Murata, Takashi Kuroda, and Waldemar Karwowski. 2017. Effects of auditory and tactile warning on response to visual hazards under a noisy environment Applied Ergonomics 60 (2017), 58-67. https://doi.org/10.1016/j.apergo.2016.11.002

[20] Takuji Narumi, Akagawa Tomohiro, Young Ah Seong, and Michitaka Hirose 2009. Characterizing the space by thermal feedback through a wearable device. Lecture Notes in Computer Science (including subseries Lecture Notes in Artificial Intelligence and Lecture Notes in Bioinformatics) 5622 LNCS (2009), 355-364. https: //doi.org/10.1007/978-3-642-02771-0\{_\}40

[21] Roshan Lalintha Peiris, Wei Peng, Zikun Chen, Liwei Chan, and Kouta Minamizawa. 2017. ThermoVR: Exploring Integrated Thermal Haptic Feedback with Head Mounted Displays Roshan. Proceedings of the 2017 CHI Conference on Human Factors in Computing Systems - CHI '17 (2017), 5452-5456. https://doi.org/10.1145/3025453.3025824

[22] Christopher J. Ploch, Jung Hwa Bae, Caitlin C. Ploch, Wendy Ju, and Mark R. Cutkosky. 2017. Comparing haptic and audio navigation cues on the road for distracted drivers with a skin stretch steering wheel. 2017 IEEE World Haptics
Conference, WHC 2017 June (2017), 448-453. https://doi.org/10.1109/WHC.2017. 7989943

[23] Ioannis Politis, Stephen Brewster, and Frank Pollick. 2013. Evaluating Multimodal Driver Displays of Varying Urgency. Proceedings of ACM AutoUI 201313 (2013), 92-99. https://doi.org/10.1145/2516540.2516543

[24] Ioannis Politis, Stephen Brewster, and Frank Pollick. 2014. Evaluating Multimodal Driver Displays under Varying Situational Urgency. Proceedings of the 32nd annual ACM conference on Human factors in computing systems - CHI '14 (2014), 4067-4076. https://doi.org/10.1145/2556288.2556988

[25] Shadan Sadeghian Borojeni, Lewis Chuang, Wilko Heuten, and Susanne Boll. 2016. Assisting Drivers with Ambient Take-Over Requests in Highly Automated Driving. Proceedings of the 8th International Conference on Automotive User Interfaces and Interactive Vehicular Applications - Automotive'UI 16 (2016), 237244. https://doi.org/10.1145/3003715.3005409

[26] Katri Salminen, Veikko Surakka, Jukka Raisamo, Jani Lylykangas, Johannes Pystynen, Roope Raisamo, Kalle Mäkelä, and Teemu Ahmaniemi. 2011. Emotional responses to thermal stimuli. Proceedings of the 13th international conference on multimodal interfaces - ICMI '11 (2011), 193. https://doi.org/10.1145/2070481.2070513

[27] J.J. Scott and Robert Gray. 2008. A Comparison of Tactile, Visual, and Auditory Warnings for Rear-End Collision Prevention in Simulated Driving. Human Factors: The fournal of the Human Factors and Ergonomics Society 50, 2 (2008), 264-275. https://doi.org/10.1518/001872008X250674

[28] Anshul Singhal and Lynette A. Jones. 2017. Perceptual interactions in thermotactile displays. 2017 IEEE World Haptics Conference, WHC 2017 June (2017), 90-95. https://doi.org/10.1109/WHC.2017.7989882

[29] Katja Suhonen, Sebastian Müller, Jussi Rantala, Kaisa Väänänen-Vainio-Mattila, Roope Raisamo, and Vuokko Lantz. 2012. Haptically augmented remote speech communication. Proceedings of the 7th Nordic Conference on Human-Computer Interaction Making Sense Through Design - NordiCHI '12 (2012), 361. https: //doi.org/10.1145/2399016.2399073

[30] Keisuke Suzuki and Håkan Jansson. 2003. An analysis of driver's steering behaviour during auditory or haptic warnings for the designing of lane departure warning system. FSAE Review 24, 1 (2003), 65-70. https://doi.org/10.1016/S03894304(02)00247-3

[31] Graham Wilson and Stephen Brewster. 2017. Multi-Moji: Combining Thermal, Vibrotactile \& Visual Stimuli to Expand the Affective Range of Feedback. Proceedings of the 2017 CHI Conference on Human Factors in Computing Systems CHI '17 (2017), 1743-1755. https://doi.org/10.1145/3025453.3025614

[32] Graham Wilson, Stephen Brewster, Martin Halvey, and Stephen Hughes. 2012. Thermal Icons: Evaluating Structured Thermal Feedback for Mobile Interaction. Proceedings of the 14th international conference on Human-computer interaction with mobile devices and services - MobileHCI '12 (2012), 309. https://doi.org/10. 1145/2371574.2371621

[33] Graham Wilson, Dobromir Dobrev, and Stephen Brewster. 2017. Hot Under the Collar: Mapping Thermal Feedback to Dimensional Models of Emotion. Proceedings of the 2017 ACM Workshop on Interacting with Smart Objects - SmartObject '17 (2017), 31-36. https://doi.org/10.1145/3038450.3038454

[34] Graham Wilson, Martin Halvey, Stephen Brewster, and Stephen A. Hughes. 2011. Some Like it Hot? Thermal Feedback for Mobile Devices. Proceedings of the 2011 annual conference on Human factors in computing systems - CHI '11 (2011), 2555. https://doi.org/10.1145/1978942.1979316

[35] Graham Wilson, Harry Maxwell, and Mike Just. 2017. Everything's Cool: Extending Security Warnings with Thermal Feedback. In Proceedings of the 2017 CHI Conference Extended Abstracts on Human Factors in Computing Systems - CHI EA '17. ACM, Denver, Colorado, USA, 2232-2239. https://doi.org/10.1145/3027063. 3053127 